\title{
Dimensional crossover and quantum effects of gases adsorbed on nanotube bundles
}

\author{
M. Mercedes Calbi and Milton W. Cole \\ Physics Department, \\ Pennsylvania State University, \\ University Park, \\ Pennsylvania 16802
}

(Dated: November 20, 2018)

\begin{abstract}
Adsorption properties of several gases $\left(\mathrm{Ne}, \mathrm{CH}_{4}, \mathrm{Ar}, \mathrm{Xe}\right)$ on the external surface of a carbon nanotube bundle are investigated. Calculations are performed at low coverage and variable temperature, and for some fixed temperatures as a function of coverage. Within a simple model (in the limit of very low coverage) we are able to study the evolution of the film's thermal properties from those of a one dimensional (1D) fluid to those of a 2D film. In addition, grand canonical Monte Carlo simulations are performed in order to identify a second layer groove phase, which occurs once a monolayer of atoms covers the external surface. We derive from the simulations the isosteric heat, compresibility and specific heat as a function of coverage. We evaluate alternative models in order to derive quantum corrections to the classical results. We compare our findings with those of recent adsorption experiments.
\end{abstract}




\section{INTRODUCTION}

The nature of gas adsorption within and outside of bundles of carbon nanotubes is a

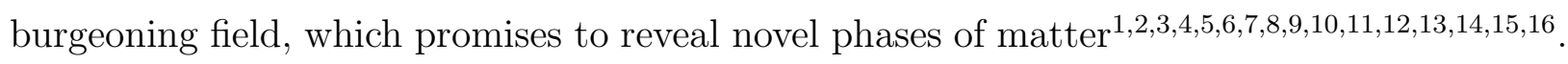
Several recent experiments have explored the behavior of gases adsorbed on the outside of bundles, or ropes, consisting of many nanotubes having nearly parallel orientation. Our group has studied this system with classical computer simulation (in the case of $\mathrm{Ne}, \mathrm{Ar}, \mathrm{Kr}$ and $\mathrm{Xe}$ ) and model ground state calculations purporting to describe this external surface adsorption of classical, or nearly classical, gases 17,18 . Other studies, both experimental and theoretical, have been undertaken of the behavior of quantum gases, such as hydrogen or helium, in quasi-one dimensional (1D) models of this environment国昍时.

Figure 1 shows the potential energy $V(\mathbf{r})$ of a methane molecule on the external surface of a nanotube bundle, computed (as discussed in Section IV) by summing empirical LennardJones pair interactions between the molecule and the $\mathrm{C}$ atoms comprising the nanotube (which are actually smeared out to form "continuum carbon" on a cylindrical surface) $1 \mathrm{~g}$. The characteristic features of $\mathrm{V}$ are a small region nestled between tubes, called a "groove", in which the potential is extremely attractive, and a more extended region where the potential varies only slowly along the surface. It is not surprising that the adsorption at a given temperature (computed in Section IV) exhibits a 1D fluid regime, confined to the groove, and a 2D monolayer regime as the number of adsorbed particles increases. The evolution of this behavior as a function of gas pressure $(\mathrm{P})$ and temperature $(\mathrm{T})$ is qualitatively the same as that found in our simulations of the other gases 17.18 .

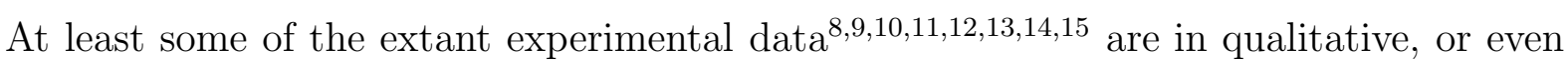
quantitative, agreement with calculations based on such simple model assumptions about the geometry (nanotubes are uniform, identical and parallel to one another) and interactions (pairwise additive)20. As an example, we state in Table I the ratio between the binding energies in the groove and on graphite in the low density limit, obtained from our calculations and two different experimental groups. Thereby encouraged, we proceed in the present paper to address several aspects of this adsorption problem that were either ignored in our previous studies or received only cursory attention there. One particular goal is to better understand how the film's thermal properties evolve from those of an essentially 1D fluid to those of a monolayer (and eventually bilayer) film adsorbed on this bundle's surface. An 
aspect of this investigation is to identify and characterize a "second layer groove phase", for which simulations and experiments have provided some evidence 10 17. 12 . A second principal goal of the present study is to understand how quantum effects alter the thermodynamic quantities of interest. These effects are particularly important for $\mathrm{H}_{2}, \mathrm{He}, \mathrm{Ne}$ and $\mathrm{CH}_{4}$ films in the present geometryt. We emphasize that the potentials we use, like all semiempirical potentials, are uncertain to some degree. As an example, we may compare the well depths computed with the present parameters and those resulting from a recent selection of "best values"21. The well depth from a single graphene sheet is given by $D_{\text {graphene }}=(6 \pi / 5) \epsilon_{g c} \sigma_{g c}^{2} \theta_{c}$, where $\epsilon_{g c}$ and $\sigma_{g c}$ are the gas-carbon Lennard-Jones parameters and $\theta_{c}$ is the surface density of carbon atoms. The ratio of this depth computed with the "best" parameters to that obtained from our parameters is $0.8,0.8,1.0,1.0,1.1,1.1$ for the sequence He, Ne, Ar, Kr, Xe and $\mathrm{H}_{2}$, respectively.

In Section II, we consider the behavior of a film at very low coverage, in which case the adsorption may be considered as a single particle problem; this corresponds to the socalled "Henry's law regime" of adsorption22, in which the coverage $\mathrm{N}$ is proportional to the pressure, as specified by the Henry's law constant $\mathrm{K}_{H}=\lim _{N \rightarrow 0}(N / P)$. This "constant" typically exhibits an Arrhenius dependence on $\mathrm{T}$, with characteristic activation energy of order the heat of adsorption. As is known from analogous treatments of adsorption on planar surfaces, the $\mathrm{T}$ dependence of $\mathrm{K}_{H}$ is a sensitive function of the potential provided by the substrate, a subject of obvious interest21. We compute in this low coverage regime the specific heat (per particle) $C / N$, where $C$ is the heat capacity, and the isosteric heat

$$
q_{s t}=-\left(\frac{\partial \ln P}{\partial \beta}\right)_{N}
$$

Here $\beta^{-1}=k_{B} T$. Further, in Section II, we develop a simple "crossover potential" model, aimed at describing the film's behavior in the regime of crossover from 1D to 2D (here, a consequence of increasing T). The model works well in characterizing the thermal properties of a low coverage classical film, as demonstrated by consistency between the results from the model and those from the "exact" simulations. This agreement provides some justification for using the model to perform quantum calculations for this low coverage regime. The primary result of this calculation is a determination of the quantum correction to the thermal properties computed in the simulations. 
Section III develops a phonon model for the high density 1D fluid regime of adsorption within the groove. The treatment is a straightforward extension of the familiar Debye model used to treat bulk solids. There is an underlying premise- that phonons are, indeed, the relevant thermal excitations of a 1D fluid (since there is no crystallization), from which the thermal properties may be calculated. The reliability of this ansatz is discussed and a heuristic argument is provided to justify its use here.

Section IV presents new classical simulation results for $q_{s t}, C$ and the compressibility $(\partial N / \partial \mu)_{T}$, where $\mu$ is the chemical potential. Methane adsorption isotherms are presented for several temperatures. A key question of interest is whether the hypothetical second layer groove phase exists for all of the adsorbates we have considered, or just for $\mathrm{Ne}$, as we originally conjectured. In addressing this issue, we make contact with a growing body of

experimental data10 11. Section V summarizes our results and discusses other issues, such as the potential role of heterogeneity, which is certainly present, but is ignored in virtually all calculations to date.

\section{LOW DENSITY REGIME}

At very low density, one may neglect both interactions and quantum statistics in evaluating the film's properties. This means that the physical behavior of the system reflects the dynamics of single particles in the external field of the surface. Let $g(E)$ be the surface single particle density of states, which may be derived, in principle, by solving the Schrodinger equation in this potential field. Then, the total number of adsorbed particles at $\mathrm{T}$ and $\mu$ satisfies

$$
N=e^{\beta \mu} \int d E g(E) e^{-\beta E}=e^{\beta \mu} Q(\beta)
$$

Here $Q(\beta)$ is the single particle partition function. For an ideal spinless 23 3D gas, assumed to be in equilibrium with the film, $e^{\beta \mu}=\beta \lambda_{T}^{3} P$, where $\lambda_{T}$ is the de Broglie thermal wavelength $\left(\lambda_{T}^{2}=2 \pi \beta \hbar^{2} / m\right)$. By differentiating Eq. 2, at fixed $\mathrm{N}$, we obtain the quantum expression for the isosteric heat at low density:

$$
q_{s t}=\frac{5}{2 \beta}-\langle E\rangle=\frac{5}{2 \beta}+\left(\frac{\partial \ln Q}{\partial \beta}\right)_{N, A}
$$


$\langle E\rangle$ is the quantum-statistical average of the energy per particle. The quantum value of the Henry's law coefficient satisfies

$$
K_{H}=\beta \lambda_{T}^{3} Q(\beta)
$$

The quantum specific heat per particle (expressed in units of Boltzmann's constant) at fixed surface area $A$ satisfies

$$
\frac{C}{N k_{B}}=\beta^{2}\left(\frac{\partial^{2} \ln Q}{\partial \beta^{2}}\right)_{N, A}
$$

and from Eq. 3,

$$
\frac{1}{k_{B}} \frac{d q_{s t}}{d T}=\frac{5}{2}-\frac{C}{N k_{B}}
$$

Classically, one employs an alternative formulation of the behavior of an ideal gas in the external field of the substrate. The Henry's law constant is then

$$
K_{H}^{c l}=\beta \int d \mathbf{r} e^{-\beta V(\mathbf{r})}=\beta Z(\beta)
$$

$Z(\beta)$ is a classical single particle configuration integral and the integration domain is a (somewhat arbitrarily defined) volume near the substrate. The classical isosteric heat differs from the quantum expression because the quantum mean energy is replaced by the classical energy, $\frac{3}{2 \beta}+\langle V\rangle_{c l}$ :

$$
q_{c l}=\frac{1}{\beta}-\langle V\rangle_{c l}=\frac{1}{\beta}+\frac{d \ln Z}{d \beta}
$$

The classical specific heat is given by

$$
\frac{C_{c l}}{N k_{B}}=\frac{3}{2}+\beta^{2} \frac{\partial^{2} \ln Z}{\partial \beta^{2}}
$$

For reference, we present in the Appendix analytical results for the limiting case of motion in $1 \mathrm{D}$ with a quadratic transverse confining potential

$$
V(r)=V_{0}+\alpha \frac{r^{2}}{2}
$$

where $r^{2}=x^{2}+y^{2}$ and the $z$ axis is the usual cylindrical symmetry axis, coincident with the groove site. 
The full line in Fig.2 depicts the classical specific heat in the limit of zero coverage computed from the potential energy shown in Fig.1. One observes that the behavior crosses over from the $1 \mathrm{D}$ value $(5 / 2$, as shown in the Appendix) at low $\mathrm{T}$ to a value near the $2 \mathrm{D}$ value (2, since $y$ motion is included) at high $\mathrm{T}$. However, there appears a dramatic peak at intermediate $\mathrm{T} \approx 200 \mathrm{~K}$. This behavior can be explained qualitatively, as follows.

We invoke a simple "crossover model" of the potential which divides configuration space into two regions, giving additive contributions to the partition function:

$$
Z(\beta)=\int_{\text {groove }} d^{2} r e^{-\beta\left(V_{g}+\frac{1}{2} \alpha r^{2}\right)}+L_{s} \int_{\text {mono }} d y e^{-\beta\left(V_{m}+\frac{1}{2} k_{m} y^{2}\right)}
$$

The first integral is a $2 \mathrm{D}$ integral over the groove region, where we assume the potential energy to be of harmonic type. The second integral evaluates the contribution from the monolayer region over the external surface of the tubes, assuming a harmonic potential along the direction perpendicular to the surface. $L_{s}$ is the length of this region (a fraction of $2 \pi R, R$ being the radius of the tubes) over which the monolayer states can extend. We estimate reasonable values for each constant in the model (radius of the groove region $\approx 1 \AA$, $V_{g}=-2028 \mathrm{~K}, \alpha=4800 \mathrm{~K} / \AA^{2}, V_{m}=-1050 \mathrm{~K}, k_{m}=6000 \mathrm{~K} / \AA^{2}, L_{s}=18 \AA$ ) from the full potential of Fig. 1. Using this model partition function, we obtain the results shown in Fig. 2 as a thin line. The position and shape of the peak in Fig. 2 agree well with the "exact" results. This implies that the model has the key physical ingredient, which is the crossover from $1 \mathrm{D}$ to $2 \mathrm{D}$ regimes. We observe that the peak temperature $(\approx 220 \mathrm{~K})$ is significantly less than the energy difference between monolayer and groove potentials $\left(V_{m}-V_{g} \approx 1000\right.$ $\mathrm{K})$.

However, we note qualitative differences between the "exact" and model curves at both low and high T. These differences come from the deviation of the full potential from the model's assumed harmonic shape. First, we consider the linear region of variation of $\mathrm{C}$ at low T. This behavior may be derived from perturbation theory, assuming that there is a "small" quartic term in the potential, $\delta V$, which may be expressed in terms of appropriate coefficients $a_{i}$ :

$$
\delta V(x, y)=a_{1} x^{2} y^{2}+a_{2}\left(x^{4}+y^{4}\right)
$$

The classical energy shift $\delta E$ is just the expectation value of the perturbation (evaluated 
with the harmonic potential, Eq.10); this leads to $24 \delta E=\left(a_{1}+6 a_{2}\right) /(\alpha \beta)^{2}$. This quartic correction to $E$ gives rise to terms proportional to $\mathrm{T}$ in the specific heat that are observed in Fig. 2 at low T, i.e. the regime $(T<100 \mathrm{~K})$ where the quartic terms are adequately described by perturbation theory. At very high $\mathrm{T}$, the deviation of $C /\left(N k_{B}\right)$ from the $2 \mathrm{D}$ value (2) occurs because of the large $y$ behavior of the full potential, which lies below the harmonic approximation.

The simple crossover model potential is thus seen to yield a specific heat which agrees qualitatively with the numerical results (without fitting the parameters). Hence, we conclude that the model contains the essential ingredients possessed by the "true" potential. We now use the simple model potential in another way to deduce the quantum expression for the specific heat and other thermal properties at very low N. To do the quantum calculation accurately requires a determination of the density of states in this inhomogeneous environment. Our alternative method involves a further set of approximations, which obviate solving the full 3D Schrodinger equation (SE). An exact solution is not necessary, in our opinion, given the uncertainty in the potential $\mathrm{V}(\mathbf{r})$ and the manifestly satisfactory character of the simple crossover model potential. To obtain the energy spectrum, we solve the SE with an adiabatic approximation, which has been shown to predict band structures for He atoms on graphite 25 . In this approximation, we first solve the SE for adatom motion perpendicular to the surface at each point $(x)$ on the surface. The eigenvalue for this $\mathrm{z}$ motion becomes an effective potential energy $\mathrm{V}_{\text {eff }}(x)$. As in the conventional treatment of diatomic molecules (focusing on the electronic ground state), we assume that the lowest state for the adatom's $z$ motion is the only energy needed for the "slower" degree of freedom, i.e. motion along the surface. Figure 3 shows the resulting effective potential as well as a fit to this potential of the following form:

$$
U(x)=V_{m}+\frac{V_{0}}{\cosh ^{2}(x / a)}
$$

The reason for this choice of model potential is that the shape is appropriate, as seen in the figure, and the spectrum is known for this functional form, as given by 6 f

$$
\epsilon_{n}=-\frac{\hbar^{2}}{2 m a^{2}}\left[\frac{1}{2} \sqrt{\frac{8 m V_{0} a^{2}}{\hbar^{2}}+1}-\left(n+\frac{1}{2}\right)\right]^{2} \quad ; n=0,1,2 \ldots
$$

The largest value that $n$ can take depends on the parameters of the model potential. For 
example, we obtain 21 discrete levels for $\mathrm{CH}_{4}, 11$ for $\mathrm{Ne}$ and 3 for $\mathrm{H}_{2}$. With this spectrum, we can factorize $Q(\beta)$ in the following way:

$$
Q(\beta)=\frac{L}{\lambda_{T}}\left[Q_{-}(\beta)+Q_{+}(\beta)\right] Q_{y}(\beta)
$$

where $L / \lambda_{T}$ is the sum of the free particle states along the $z$ direction, $Q_{y}(\beta)$ comes from the contribution of the discrete states in a potential well along the direction perpendicular to the surface (here assumed to be a harmonic oscillator's spectrum):

$$
Q_{y}(\beta)=\sum_{n} e^{-n \beta \hbar \omega_{y}}=\frac{1}{1-e^{-\beta \hbar \omega}}
$$

$\left[Q_{-}(\beta)+Q_{+}(\beta)\right]$ is the partition function of all the states in the effective potential along the $x$ direction. The first term $\left(Q_{-}\right)$comes from the discrete states in $V_{\text {eff }}(x)$ :

$$
Q_{-}(\beta)=\sum_{n} e^{-\beta \epsilon_{n}}
$$

The contribution $Q_{+}$from the continuum density of states is modeled by scaling the continuum spectrum of a uniform system in a constant potential of depth $V_{m}$ by the length of a unit cell $d$ (here equal to the horizontal separation between nanotubes):

$$
Q_{+}(\beta)=e^{-\beta V_{m}} \frac{d}{\lambda_{T}}
$$

With these ingredients, the thermodynamic properties can be computed and compared with the corresponding classical quantities, obtained above from the original potential. The dashed line in Fig. 2 show the quantum result for C. In this case, the low $\mathrm{T}$ behavior corresponds to a quantum 1D regime characterized by the excitation of only the low lying states in $V_{\text {eff }}$. Note that the zero temperature limit is 0.5 , as expected for a quantum 1D system. Deviation from that limit occurs when T comes within an order of magnitude of the transverse excitation energy in the groove $\left(\approx 90 \mathrm{~K}\right.$ for $\left.\mathrm{CH}_{4}\right)$. This behavior differs markedly from the classical result in Fig. 2. Fig. 4 shows the corresponding results for the isosteric heat. At zero $\mathrm{T}$, the classical result (full and thin lines) corresponds to the minimum of the potential in the groove while the quantum result is the ground state energy $\varepsilon_{0}$ in that potential. Another important distinction at low temperature is that quantum results imply an increase of the isosteric heat with $\mathrm{T}$, whereas classically a decrease is expected. We observe that $q_{s t}=-\varepsilon_{0}+2 k_{B} T$ when $T<50 \mathrm{~K}$ and it reaches its maximum value when 
$C /\left(N k_{B}\right)=5 / 2($ Eq. 6$)$ at $T \approx 100 \mathrm{~K}$. As shown in the Appendix, at low $\mathrm{T}$ the difference between quantum and classical heats is $\delta q_{s t}=2 / \beta-\hbar \omega$, where $\hbar \omega$ is the zero point energy in the groove. At high $\mathrm{T}$, the temperature dependence of $q_{s t}$ is given by $k_{B} T / 2$, which corresponds to 2D motion confined by a transverse harmonic potential. Figure 5 (a) and (b) display the results for $\mathrm{C}$ and $q_{s t}$ in the case of Ne. We notice that the crossover occurs at a much lower $\mathrm{T}(\approx 100 \mathrm{~K})$ for $\mathrm{Ne}$ than for $\mathrm{CH}_{4}$.

In closing this section concerning low density, we note that the first order correction due to quantum statistics can be determined in a straightforward way, permitting an assessment of its importance in the analysis. To do this, one expands the exact equation relating $\mathrm{N}$ and $\mu$, assuming that the fugacity $e^{\beta \mu}$ is small; this is the usual way to develop a quantum virial expansion for translationally invariant systems. The result in the present case is

$$
N=Q(\beta) e^{\beta \mu}[1 \pm f(N, \beta) \ldots]
$$

The first term leads to the classical regime addressed above; the "correction" term $f$ (negative/positive for fermions/bosons) in brackets becomes:

$$
f(N, \beta)=\frac{Q(2 \beta)}{Q^{2}(\beta)}=\frac{\rho \lambda_{T}}{\sqrt{2}}\left(\frac{1-e^{-b}}{1-e^{-2 b}}\right)^{2}
$$

Here $b=\beta \hbar \omega$. The factor preceding the expression in parentheses is analogous to quantum statistical correction terms found in $2 \mathrm{D}$ and $3 \mathrm{D}$ expansions. We observe that the classical approximation used earlier is appropriate when the interparticle spacing exceeds $\lambda_{T}$ (the same constraint as that found in the analogous $2 \mathrm{D}$ and 3D problems). The factor in parentheses in Eq. 20 is always less than one, so it helps the statistical expansion to converge, especially at high $\mathrm{T}$ when the factor becomes $1 / 4$. The reason for this reduced statistical correction is simply that the crowding in phase space, the origin of effects of quantum statistics, is reduced by a spreading among the many transverse states that are excited when $b<1$. Finally, we note that statistical corrections to the noninteracting classical gas are relatively more important in $1 \mathrm{D}$ than in higher dimensions $\mathrm{D}$. The reason is that these corrections appear as products of density and $\lambda_{T}^{d}$. At a given $\mathrm{T}$, therefore, the effect of statistics appears at a lower $\mathrm{T}$ in a system of lower $\mathrm{D}$ (all other things being equal). 


\section{PHONONS IN THE HIGH DENSITY GROOVE PHASE}

The quasi-1D fluid within the groove represents a novel system in many ways. One is that the low-lying excitations of the system are expected to be phonons, even in the absence of a crystal (which is assumed in the usual derivation of phonons in terms of an expansion in displacements from equilibrium). Such phonons must exist as longitudinal, long wavelength excitations of this system, derived from elasticity theory or quantum hydrodynamics (as in the liquid helium case)27. Their speed for a nearly classical system can be derived from the classical equation of state, $m c^{2}=(d P / d \rho)_{T}$. Such data appear in Fig. 6. One observes a minimum in $c$ at density corresponding to the incipient condensation at $\mathrm{T}=0$.

We analyze the behavior using the Debye model, applied to a fluid of density $\rho$ and sound speed $c$. The Debye wave vector and frequency are $k_{D}=\pi \rho$ and $\omega_{D}=c k_{D}$, respectively. The conventional 3D treatment is changed to accommodate the $1 \mathrm{D}$ density of states, which is a constant below $\omega_{D}$ :

$$
N(\omega)=\frac{L}{\pi c} \Theta\left(\omega-\omega_{D}\right)
$$

Here $\Theta(x)$ is the Heaviside unit step function. The resulting thermal energy per unit length is

$$
\frac{E}{L}=\frac{1}{\pi c \beta^{2} \hbar} \int_{0}^{x_{m}} d x \frac{x}{e^{x}-1}
$$

The upper limit to the integral arises from the Debye frequency cutoff, $x_{m}=\beta \hbar \omega_{D}$. At high T, $x_{m}<<1$, this expression yields the $1 \mathrm{D}$ version of the law of Dulong and Petit, $C \rightarrow N k_{B}$; there is energy $k_{B} T$ per atom in this limit. At low $\mathrm{T}$, instead, the specific heat is linear in $\mathrm{T}$ :

$$
\frac{C}{N k_{B}}=\frac{\pi^{2} k_{B} T}{3 \hbar \omega_{D}}
$$

A more realistic model would yield a different numerical coefficient but the linear dependence of $\mathrm{C}$ on $\mathrm{T}$ is a robust prediction of the phonon model. The prediction in the high $\mathrm{T}$ limit is also robust, within the harmonic expansion.

One may develop a concrete realization of this behavior from the usual phonon theory. In its simplest form, we employ a model in which only nearest neighbors interact. In this 
case, the ground state corresponds to a lattice constant $a$ equal to the minimum in the pair potential. The conventional theory yields a dispersion relation

$$
\omega_{q}=2 \omega_{0} \sin \left(\frac{q a}{2}\right) \quad, \quad \omega_{0}=\sqrt{\frac{k}{m}}
$$

The force constant $k$ is just the second derivative of the pair potential, evaluated at its minimum (if the adsorbate is not compressed). If we assume a Lennard-Jones interaction $U(r)=4 \varepsilon\left[(\sigma / r)^{12}-(\sigma / r)^{6}\right]$, then the uncompressed force constant is $k_{0}=2^{8 / 3}\left(\frac{9 \varepsilon}{\sigma^{2}}\right)$. This yields a speed of sound $c=a \omega_{0}=a \sqrt{k_{0} / m}$, which depends in an explicit way on the specific system's parameters.

How important are the quantum corrections to the equation of state of the system? One measure of this is the ratio of the system's ground state zero-point energy (per atom) $E_{z p}$ to its potential energy $(\epsilon)$; this ratio is zero for a classical system. From the phonon model, we may evaluate $E_{z p}$ by summing $\hbar w_{q} / 2$ over all of the phonon modes within the Brillouin zone $(|q|<\pi / a)$. The result of this calculation is

$$
E_{z p}=\left(\frac{2}{\pi}\right) \hbar \omega_{0}
$$

Hence, the ratio of interest is

$$
\frac{E_{z p}}{\epsilon}=3\left(\frac{2^{4 / 3}}{\pi^{2}}\right) \Lambda^{*}
$$

Here $\Lambda^{*}=h /(\sigma \sqrt{m \epsilon})$ is the de Boer quantum parameter and the numerical coefficient is about 0.8. Thus, the ratio of $E_{z p}$ to $\epsilon$ is about 1.6 for $\mathrm{H}_{2}, 0.2$ for $\mathrm{CH}_{4}$ and 0.05 for Xe, assuming "typical" values of the interaction parameters. This number is indicative of the relative importance of quantum effects at low $\mathrm{T}$; the range of $\mathrm{T}$ over which quantum effects are relevant is of order this ratio times $\epsilon$. The fully classical regime is thus T exceeding 60 $\mathrm{K}, 35 \mathrm{~K}$ and $15 \mathrm{~K}$ for $\mathrm{H}_{2}, \mathrm{CH}_{4}$ and $\mathrm{Xe}$, respectively.

The preceding discussion pertains to the longitudinal phonons in the groove. There are, in addition, transverse phonons associated with motion perpendicular to the groove axis. We show here how these become important at high density, indicative of an incipient instability of the 1D state. The transverse modes (two polarizations for each wave vector) can be derived in the usual way by taking account of coupled motion of the adsorbate, as affected by the presence of the potential confining the particles within the groove $\left(\alpha r^{2} / 2\right)$. Consider 
a mode polarized in the $x$ direction and assume small amplitude displacements $\left\{x_{i}\right\}$ so that the interparticle force can be expanded about the equilibrium spacing $a$; for a given pair, the potential energy is thus shifted by

$$
V(\Delta x)-V_{0}=V^{\prime}\left[\frac{\Delta x^{2}}{2 a}\right]=f \frac{\Delta x^{2}}{2}
$$

Here, $V_{0}$ is the equilibrium spacing potential, prime means derivative with respect to the interparticle spacing, evaluated at spacing $a$, and $\Delta x$ is the difference in the $x_{i}$ values of adjacent particles. The quantity $f$ is an effective force constant associated with this coupling; $f<0$ in a highly compressed phase. Assuming only nearest neighbor interactions, the equation of motion for a mode of wave vector $q$ can be solved, yielding the transverse spectrum

$$
\omega_{t}^{2}(q)=\omega_{1}^{2}+\left(4 \frac{f}{m}\right) \sin ^{2}\left(\frac{q a}{2}\right) \quad, \quad \omega_{1}^{2}=\frac{\alpha}{m}
$$

At $q=0$, the frequency is just that $\left(\omega_{1}\right)$ of single particles in the external potential. However, the finite $q$ frequency is lower if the adsorbate is compressed. At the zone boundary, $\omega$ is a minimum in this case. There arises, therefore, an instability when

$$
f<-m \omega_{1}^{2} / 4=-\alpha / 4
$$

This condition is equivalent to the energy minimum condition, which favors a periodic structure if the density becomes high, so that the repulsive forces become too large. The preceding equation represents an instability criterion, but one expects there to be a lower density regime of dynamical stability but energetic metastability. We have explored this problem in recent work by comparing the energies of alternative structures on the external surface of the bundle 28 . We concluded that the transition in question occurs from the groove to the three-stripe phase. An alternative possibility 28, a two-stripe phase (two parallel chains of atoms), was less favored in the Lennard-Jones interaction case we have considered.

We have made a quantitative comparison with the phonon instability scenario in one case, $\mathrm{CH}_{4}$ in a groove. In that case, the transition to the three stripe phase occurs at $10 \%$ compression in lattice constant, according to the $\mathrm{T}=0$ calculations. The instability condition above corresponds to a further compression of the lattice constant by $20 \%$. Hence, the 1D 
phase becomes metastable (relative to the three-stripe phase) at considerably lower density, preempting the instability-driven transition.

In the limit of small compression, we may neglect the term in Eq. 28 proportional to $f$. In that case, the transverse motion reduces to that of independent particles. The resulting effect on the thermal properties of the adsorbate coincides with that of single particles, i.e. the low density limit, discussed in the Appendix.

\section{COVERAGE DEPENDENT ADSORPTION: GRAND CANONICAL MONTE CARLO SIMULATIONS}

As in our previous studiest 18 , we use grand canonical Monte Carlo simulations to investigate the adsorption behavior of $\mathrm{CH}_{4}$ on the external surface of a bundle. The potential energy experienced by the adsorbate particles in that region is modeled by summing the contributions from two adjacent nanotubes, by adding Lennard-Jones two body interactions (with distance and energy parameters $\sigma_{g c}=3.56 \AA, \epsilon_{g c}=67.2 \mathrm{~K}$ ) between the adsorbate particle and the nanotube's carbon atoms. The dimensions of the simulation cell are set to be $17 \AA$ in the $x$ direction (which corresponds to the center to center distance between the tubes), $10 \sigma_{g g}$ along the $z$ direction (tube's axis direction) and $40 \AA$ for the height of the box along the $y$ direction. The details of our model assumptions and the simulation method can be found in Ref. 17.

Figure 7 shows the resulting $\mathrm{CH}_{4}$ adsorption isotherms at various temperatures. Since the length of the cell in the $z$ direction is $10 \sigma_{g g}$, the saturation number of atoms in a single line (closed packed) is $\langle N\rangle=9$. The phase behavior evolves with $\mu$ in the same manner observed for other gases 17 , starting with a line of atoms in the groove at very low pressure. At low $\mathrm{T}$, a step (not noticeable for the temperatures shown in the figure) appears to a socalled "3-stripe" phase, consisting of two additional lines of atoms parallel to that in the groove. At higher pressure a monolayer $(\langle N\rangle \approx 45,5$ lines $)$ is formed over the external surface. Once it completes, there appears some evidence of a transition to a second layer groove phase $(\langle N\rangle \approx 54)$, i.e. a single line of atoms formed above the monolayer phase, in the new groove region. This phase was observed in our previous simulations in the case of $\mathrm{Ne}, \mathrm{Ar}$ and $\mathrm{Kr}$ and also experimentally in recent work of Migone's group 10 . 1 . Subsequent transitions to bilayer and three-layer phases are observed as the pressure increases. 
In figure 8 we display the evolution of the density projected onto the transverse $x y$ plane at $T=90 \mathrm{~K}$ as the coverage increases, starting at the monolayer phase. We observe the formation of the second layer groove, a dilute second layer 3-stripe phase and the completion of the second layer. The variation of potential along the second layer surface is still large enough to gives rise to the appearance of a third-layer groove. After that, the adsorbate surface has considerably flattened at this point, causing the subsequent film to be much less structured.

We consider now the formation of the second layer groove phase for three different gases of increasing size: $\mathrm{Ne}, \mathrm{CH}_{4}$ and Xe. Figure 9 shows its respective isotherms for high coverage. The arrows point the appearance of the second layer groove. Table II displays the values of the pressure from the isotherms at which this phase occurs in comparison with the experimental values1 11 , indicating a very good agreement. To identify this phase we also show the corresponding density contours (Fig. 10) and the compresibility $d N / d \mu$ (Fig. 11) calculated from the number fluctuation in the simulations:

$$
\frac{d N}{d \mu}=\frac{\left\langle(\Delta N)^{2}\right\rangle}{k_{B} T}
$$

Here $\left\langle(\Delta N)^{2}\right\rangle$ is the variance in the number of particles in the simulation (at fixed $\mu$ and $\mathrm{T})$. For the smallest atom, Ne, there occurs a peak near $\langle N\rangle \approx 80$, which corresponds to the (best-defined) second layer groove phase. Then, transitions in the second layer (three-stripe phase and completion of the second layer) cause the appearance of a second broader peak at $\langle N\rangle \approx 100-110$, but they cannot be distinguished individually. For $\mathrm{CH}_{4}$, there is a smaller peak near $\langle N\rangle \approx 60$ (second layer groove) but it merges with the peak corresponding to the second layer transition $(\langle N\rangle \approx 70)$. Something similar happens in the case of Xe. The peak starts with the second layer groove transition $(\langle N\rangle \approx 55)$ but it immediatly continues with the bilayer transition $(\langle N\rangle \approx 80)$.

Another quantity which should show the phase transitions as the coverage increases is the isosteric heat. Figure 12.(a) shows the isosteric heat as a function of linear coverage computed from adsorption isotherms of the different gases. The region $\rho \sigma_{g g}<1$ is the groove-filling region. Once the groove is filled, the isosteric heat decreases abruptly due to the high energy difference between this site and the surface site. The following decrease is observed at the monolayer completion but before that a small increase strongly suggests the presence of the second layer groove. 
In Tables III and IV, we compare the isosteric heat values corresponding to the first groove phase and the monolayer phase with the available experimental results from two different groups. We observe that the agreement is quite good.

In Fig. 12.(b) we compare the whole coverage dependence of the isosteric heat for Ar derived from our simulations with the experimental result, 13 . We observe that the general trend is qualitatively similar.

Fig 13 shows the specific heat as a function of coverage for $\mathrm{Kr}$ and $\mathrm{Ar}$ calculated from the simulations data. In both cases, a notable increase is observed near the completion of the groove and a smaller one is present at the end of the monolayer completion, very possibly due to the presence of the second groove phase. The low density value $(N<5)$ agrees reasonably well with the low density limit calculations of Section II for those gases and temperatures.

\section{SUMMARY AND CONCLUSIONS}

Our calculations have yielded classical and quantum behavior of diverse gases adsorbed on the external surface of a nanotubes bundle. The present results for thermal properties are to be supplemented, in general, by contributions from gases adsorbed in other sites, i.e. the interstitial channel and endohedral positions, if these are accessible to the adsorbate. The degree to which this is the case appears to be very sensitive to sample preparation and purification technique. Evidence in this paper's tables provides some tentative support for the belief that a significant fraction of these samples' area is ordered and clean. Yet Fig.12(b) shows a qualitative discrepancy, presumably attributable to heterogeneity.

We have explored the problem of dimensional crossover by studying adsorption along two distinct thermodynamic paths: constant $\mathrm{T}$ (variable $\mathrm{N}$ ) and variable $\mathrm{T}$ (very low $\mathrm{N}$ ). At very low coverage, the effective dimensionality increases progressively with $\mathrm{T}$ because the adsorbed molecules migrate over a T-dependent phase space, beginning (at low $\mathrm{T}$ ) with the groove and ending with monolayer and even 3D regimes at higher T. Qualitatively similar

evolution was investigated some years ago in the case of He isotopes on graphite2 2 . In that case, the $\mathrm{T}$ dependence of the dimensionality (calculated and measured) reflects the energy dependence of the wave functions' spatial localization. The more conventional method of studying dimensional crossover is to assess the variation with coverage of film structure 
and thermal properties. This is the route followed in adsorption isotherm measurements. Evidently, one can (in principle) explore the N-T plane along any path. An interesting question that we have not explored is how the effective dimensionality of the film varies at higher $\mathrm{T}$ or $\mathrm{N}$ than is reported here, yielding a more complete characterization of the effective dimensionality. We note that specific heat and isotherm experiments provide complementary information, so that both experiments are worth carrying out 30 . To our knowledge, no specific heat measurements have yet been undertaken for gas adsorption on nanotubes. This situation is probably temporary, because the very high specific area found in nanotubes samples should yield very high total heat capacities, with a relatively small background correction at low T. This argument suggests that measurement and interpretation of C(N,T) data are likely to be fruitful and convenient.

Our computational methods used in this paper are relatively straightforward, i.e. mostly extensions of those used in our previous simulation studies. Hence, we have found few surprises in the results. One of the most intriguing findings is that the second layer groove phase is present in the isotherms (dramatically so in the compressibility) for all of the systems studied, consistent with experiments of the Migone group. Equally encouraging is the agreement reported in the previous section between these calculations and experimentally observed thermodynamic quantities. Such consistency is initially surprising, in view of the simplified potential models. One concludes that the interaction strengths are adequately transferable from the graphite adsorption problem. Such behavior is not consistent with some model calculations in which either curvature-induced distortion of the physisorption potential or sensitivity of the potential to the nanotubes' conductivity is present.

\section{Acknowledgments}

We wish to acknowledge Mary Jo Bojan, Bill Steele, Silvina Gatica, Milen Kostov, Aldo Migone, J.G. Dash, Karl Johnson, Oscar Vilches and Michel Bienfait for stimulating discussions. We are grateful to the Petroleum Research Foundation of the American Chemical Society, the Army Research Office and Fundación Antorchas for their support. 


\section{APPENDIX A: 1D MOTION}

In the limiting case of motion in $1 \mathrm{D}$ with a quadratic transverse confining potential

$$
V(r)=V_{0}+\alpha \frac{r^{2}}{2}
$$

where $r^{2}=x^{2}+y^{2}$ and the $z$ axis is the usual cylindrical symmetry axis, the classical configuration integral satisfies

$$
Z(\beta)=\frac{2 \pi L}{\beta \alpha} e^{-\beta V_{0}}
$$

From Eq.8 and 9, classical values of the thermal variables in this quasi-1D limit are

$$
\begin{aligned}
q_{c l} & =-V_{0} \\
\frac{C_{c l}}{N k_{B}} & =\frac{5}{2} \\
\mu_{c l} & =V_{0}+\frac{1}{\beta} \ln \left(\frac{\rho \lambda_{T}}{\pi} \frac{\lambda_{T}^{2}}{\left\langle r^{2}\right\rangle}\right)
\end{aligned}
$$

where $\left\langle r^{2}\right\rangle=2 /(\alpha \beta)$ is the mean square particle displacement perpendicular to the axis. The analogous quasi-1D quantum values are given by assuming that the temperature is sufficiently low that only the lowest transverse vibration is present, for which the energy is $\varepsilon_{0}=V_{0}+\hbar \omega, \omega=\sqrt{\alpha / m}$.

In this case, $g(E)=G_{1}\left(E-\varepsilon_{0}\right) \Theta\left(E-\varepsilon_{0}\right)$, where $G_{1}(x)=\frac{L}{\hbar \pi} \sqrt{\frac{m}{2 x}}$. This is the $1 \mathrm{D}$ density of states for a hypothetical system with no transverse degrees of freedom. Then, the single particle partition function is

$$
Q(\beta)=\frac{L}{\lambda} e^{-\beta \varepsilon_{0}}
$$

A generalization of this expression to include all transverse states, within the harmonic approximation, leads to correction to $Q$ by a factor, $\left(1-e^{-\beta \hbar \omega}\right)^{-2}$. Without these factors, 


$$
\begin{aligned}
\frac{C}{N k_{B} T} & =\frac{1}{2} \\
q_{s t} & =\frac{2}{\beta}-\varepsilon_{0} \\
\mu & =\varepsilon_{0}+\frac{1}{\beta} \ln (\rho \lambda)
\end{aligned}
$$

Note that the quantum isosteric heat exceeds the classical value by an amount $\delta q_{s t}$ given, in the present approximation, by $\delta q_{s t}=q_{s t}-q_{c l}=2 / \beta-\hbar \omega$. This is, as expected, the difference in energy associated with the quantized harmonic motion in $\mathrm{x}$ and $\mathrm{y}$ directions. We discuss in Section III the more general case, when other transverse degrees of freedom are excited. The present result applies to the low $\mathrm{T}$ regime, where $\hbar \omega>>2 / \beta$. 


\begin{tabular}{|c|c|c|c|}
\hline \hline & Theory & Exp.1 & Exp.2 \\
\hline $\mathrm{H}_{2}$ & 1.43 & 1.5 & 1.5 \\
\hline $\mathrm{D}_{2}$ & 1.45 & - & 1.8 \\
\hline $\mathrm{Ne}$ & 1.51 & 1.73 & - \\
\hline $\mathrm{CH}_{4}$ & 1.43 & 1.76 & 1.34 \\
\hline $\mathrm{Xe}$ & 1.41 & 1.74 & 1.37 \\
\hline \hline
\end{tabular}

TABLE I: Ratio of binding energy of a molecule in the groove to that on graphite. The values from theory correspond to the limit of zero coverage (single particle). The experimental values are obtained from isosteric heat values through the relation $\varepsilon_{0}=-q_{s t}+2 k_{B} T$, assuming that the adsorbed phase behaves as a 1D system in the temperature and density range explored. Exp.1 values are from Ref. 9 and Exp.2 values from Ref. $13\left(\mathrm{H}_{2}\right)$ and $14\left(\mathrm{CH}_{4}\right.$ and $\left.\mathrm{Xe}\right)$.

\begin{tabular}{|l|c|c|c|}
\hline \hline & $\mathrm{T}(\mathrm{K})$ & GCMC & Experiment \\
\hline $\mathrm{Ne}$ & 25 & -1.9 & - \\
\hline $\mathrm{CH}_{4}$ & 70 & -3.4 & -3.2 \\
\hline $\mathrm{Xe}$ & 112 & -2.4 & -2.6 \\
\hline \hline
\end{tabular}

TABLE II: Common logarithm of the pressure (atm) at which the second groove phase appears. The experimental values are from Ref. 11 .

\begin{tabular}{|c|c|c|c|c|c|}
\hline \hline & $\mathrm{T}(\mathrm{K})$ & $\epsilon_{g g}(\mathrm{~K})$ & GCMC & Exp.1 & Exp.2 \\
\hline $\mathrm{Ar}$ & 90 & 120 & 13.9 & - & 15.2 \\
\hline $\mathrm{CH}_{4}$ & 90 & 161 & 12.4 & 10.3 & 13.5 \\
\hline $\mathrm{Xe}$ & 110 & 221 & 12.4 & 12.5 & - \\
\hline \hline
\end{tabular}

TABLE III: Isosteric heat $q_{s t} / \epsilon_{g g}$ at a typical first groove coverage. Exp.1 values are from Ref. 8 $\left(\mathrm{CH}_{4}\right)$ and $10(\mathrm{Xe})$. Exp.2 values are taken from Ref. $13(\mathrm{Ar})$ and $14\left(\mathrm{CH}_{4}\right)$. 


\begin{tabular}{|c|c|c|c|c|c|}
\hline \hline & $\mathrm{T}(\mathrm{K})$ & $\epsilon_{g g}(\mathrm{~K})$ & GCMC & Exp.1 & Exp.2 \\
\hline $\mathrm{Ar}$ & 90 & 120 & 10.0 & - & 10.1 \\
\hline $\mathrm{CH}_{4}$ & 90 & 161 & 9.3 & 9.0 & 8.3 \\
\hline $\mathrm{Xe}$ & 110 & 221 & 8.6 & 9.9 & 8.5 \\
\hline \hline
\end{tabular}

TABLE IV: Isosteric heat $q_{s t} / \epsilon_{g g}$ at a typical monolayer coverage. Exp.1 values are from Ref. 8 $\left(\mathrm{CH}_{4}\right)$ and $10(\mathrm{Xe})$. Exp.2 values are taken from Ref. $13(\mathrm{Ar})$ and $14\left(\mathrm{CH}_{4}\right.$ and $\left.\mathrm{Xe}\right)$.

1 M.M. Calbi, M.W. Cole, S.M. Gatica, M.J. Bojan and G. Stan, Rev. Mod. Phys. 73, 857 (2001).

2 K.A. Williams and P.C. Eklund, Chem. Phys. Lett. 320, 352 (2000).

3 Q. Wang, S. R. Challa, D. S. Sholl, and J. K. Johnson, Phys. Rev. Lett. 82, 956 (1999).

4 J. Boronat, M.C. Gordillo, and J. Casulleras, J. Low Temp. Phys. 126, 199 (2002); M.C. Gordillo, J. Boronat, J. Casulleras, Phys. Rev. B 65, 014503 (2002); M.C. Gordillo, J. Boronat, J. Casulleras, J. Low Temp. Phys. 121, 543 (2000); M.C. Gordillo, J. Boronat, J. Casulleras, Phys. Rev. Lett. 85, 2348 (2000).

5 M. Boninsegni, S.Y. Lee, V.H. Crespi, Phys. Rev. Lett. 86, 3360 (2001).

6 M.M. Calbi, F. Toigo, and M.W. Cole, Phys. Rev. Lett. 86, 5062 (2001).

7 Y.H. Kahng, R.B. Hallock, E. Dujardin, and T.W. Ebbesen, J. Low Temp. Phys. 126, 223 (2002); W. Teizer, R.B. Hallock, E. Dujardin, and T.W. Ebbesen, Phys. Rev. Lett. 82, 5305 (1999); erratum 84, 1844 (2000).

8 S. Talapatra, A.D. Migone, Phys. Rev. B 65, 045416 (2002).

9 A.J. Zambano , S. Talapatra, A.D. Migone, Phys. Rev. B 64, 075415 (2001); S. Talapatra, A.Z. Zambano, S.E. Weber, and A.D. Migone, Phys. Rev. Lett. 85, 138 (2000); S.E. Weber, S. Talapatra, C. Journet, A. Zambano, and A.D. Migone, Phys. Rev. B 61, 13150 (2000).

10 S. Talapatra, A.D. Migone, Phys. Rev. Lett. 87, 206106 (2001).

11 S. Talapatra, A.D. Migone, Higher Coverage Gas Adsorption on the Surface of Carbon Nanotubes: Evidence for a New Phase in the Second Layer, unpublished.

12 A. Kuznetsova, J.T. Yates, V.V. Simonyan, J.K. Johnson, C.B. Huffman, R.E. Smalley, J. Chem. Phys. 1156691 (2001); V.V. Simonyan, J.K. Johnson, A. Kuznetsova, J.T. Yates, J. 
Chem. Phys. 114, 4180 (2001); A. Kuznetsova, J.T. Yates, J. Liu, R.E. Smalley, J. Chem. Phys. 112, $9590(2000)$.

13 T. Wilson, A. Tyburski, M.R. DePies, O.E. Vilches, D. Becquet, and M. Bienfait, J. Low Temp. Phys. 126, 403 (2002).

14 M. Muris, N. Dupont-Pavlovsky, M. Bienfait, and P. Zeppenfeld, Surf. Sci. 492, 67 (2001); M. Muris, N. Dufau, M. Bienfait, N. Dupont-Pavlovsky, Y. Grillet, J.P. Palmari, Langmuir 16, 7019 (2000).

15 K. Murata, K. Kaneko, W.A. Steele, F. Kokai, K. Takahashi, D. Kasuya, M. Yudasaka, S. Iijima, Nano Letters 1, 197 (2001); T. Ohba, K. Murata, K. Kaneko, W.A. Steele, F. Kokai, K. Takahashi, D. Kasuya, M. Yudasaka, S. Iijima, Nano Letters 1, 371 (2001).

16 H. Tanaka, M. El-Merraoui, W.A. Steele, K. Kaneko, Chem. Phys. Lett. 352, 334 (2002).

17 S.M. Gatica, M.J. Bojan, G. Stan, and M.W. Cole, J. Chem. Phys. 114, 3765 (2001).

18 M.M. Calbi, S.M. Gatica, M.J. Bojan, and M.W. Cole, J. Chem. Phys. 115, 9975 (2001).

19 G. Stan and M.W. Cole, Surf. Sci. 395, 280 (1998).

20 Recent work suggests that the long range gas-gas interaction is significantly screened by carbon atoms. See M.K. Kostov, M.W. Cole, J.C. Lewis, P. Diep, J.K. Johnson, Chem. Phys. Lett. 332, 26 (2000).

21 L.W. Bruch, M.W. Cole and E. Zaremba, Physical Adsorption: Forces and Phenomena (Oxford University Press, 1997), page 66.

22 The conventional quantity used in this definition is the thermodynamic excess coverage, rather than N. In the present case of very attractive potentials, the diference between these quantities is small.

23 Spin and other internal degrees of freedom, if any, change computed quantities by an additive constant, at most, unless there is coupling to an external field. M.K. Kostov et al (J. Chem. Phys. 116, 1720, 2002), for example, found a large effect of rotational hindrance of hydrogen molecules inside grooves and interstices of nanotube bundles. Experimental support for this was found by D.G. Narehood, P.E. Sokol, P.C. Eklund, M.K. Kostov and M.W. Cole, Phys. Rev. B in press.

24 The quantum expression is $\left(a_{1}+6 a_{2}\right)\left\langle x^{2}\right\rangle^{2}$ where $\left\langle x^{2}\right\rangle=\frac{\hbar \omega}{2 \alpha} \operatorname{coth}\left(\frac{\beta \hbar \omega}{2}\right)$.

25 M.W. Cole and F. Toigo, Phys. Rev. B 23, 3914 (1981).

26 I.I. Gol'dman and V.D. Krivchenkov, Problems in Quantum Mechanics (Dover Publications, 
Inc., New York, 1993).

27 J.G. Dash, Rev. Mod. Phys. 71, 1737 (1999).

28 P. Zeppenfeld, M. Muris, M. Bienfait, N. Dupont-Pavlovsky, and M. Johnson (to be published).

29 M.W. Cole, D.R. Frankl, and D.L. Goodstein, Rev. Mod. Phys. 53, 199 (1981).

30 R.L. Elgin and D.L. Goodstein, Phys. Rev. A 9, 2657 (1974). 


\section{FIGURE CAPTIONS}

FIG. 1: Isopotential contours for a $\mathrm{CH}_{4}$ molecule on the external surface of a nanotube bundle. The contour nearest the groove $(x=y=0)$ corresponds to $V=-2000 \mathrm{~K}$.

FIG. 2: The low density specific heat, in units of Boltzmann's constant, for $\mathrm{CH}_{4}$. Full curve is the classical specific heat obtained from Eq.9 and the potential energy shown in Fig.1. Thin curve is the classical specific heat derived from the model crossover potential. Dashed curve is the quantum specific heat from Eqs.5 and 15.

FIG. 3: Effective potential energy $\mathrm{V}_{\text {eff }}(x)$ (dashed curve) obtained by solving the Schrodinger equation for the $\mathrm{CH}_{4}$ motion perpendicular to the surface at each position $x$ on the surface. The full curve is a fit to this potential using the functional form of Eq. 13 .

FIG. 4: Isosteric heat and quantum corrections for $\mathrm{CH}_{4}$ in the three cases plotted in Fig.2.

FIG. 5: (a) Same as Fig. 2 for Ne. (b) Same as Fig. 4 for Ne.

FIG. 6: Speed of sound for $\mathrm{CH}_{4}$ in the groove as a function of reduced density at different values of reduced temperature $\mathrm{T}^{*}=\mathrm{T} / \epsilon_{g g}$.

FIG. 7: $\mathrm{CH}_{4}$ adsorption isotherms at several temperatures. Saturation capacity of the groove occurs at $\langle N\rangle=9$. The monolayer coverage is $\langle N\rangle \approx 45$.

FIG. 8: Evolution of the $\mathrm{CH}_{4}$ density with $\mathrm{P}$, at $T=90 \mathrm{~K}$. From top to bottom, in the left column: monolayer phase $\left(\mathrm{P}=0.8510^{-2}\right.$ atm $)$; second layer groove phase $(\mathrm{P}=0.17$ $\left.10^{-1} \mathrm{~atm}\right)$; second layer 3 -stripe phase $\left(\mathrm{P}=0.2010^{-1} \mathrm{~atm}\right)$. In the right column: bilayer phase $\left(\mathrm{P}=0.3010^{-1} \mathrm{~atm}\right)$; third layer groove phase $\left(\mathrm{P}=0.3710^{-1} \mathrm{~atm}\right)$; multilayer phase $\left(\mathrm{P}=0.5210^{-1} \mathrm{~atm}\right)$.

FIG. 9: Different gases' isotherms showing the formation of the second layer groove phase, occurring at points indicated by arrows.

FIG. 10: Density contours projected onto the x-y plane showing the second layer groove phase for different gases. From top to bottom: $\mathrm{Ne}(\mathrm{T}=28 \mathrm{~K}, \mathrm{P}=0.06 \mathrm{~atm}), \mathrm{CH}_{4}(\mathrm{~T}=90$ $\mathrm{K}, \mathrm{P}=0.017 \mathrm{~atm})$, and $\mathrm{Xe}(\mathrm{T}=112 \mathrm{~K}, \mathrm{P}=0.005 \mathrm{~atm})$.

FIG. 11: Compressibility as a function of coverage for different gases and coverages beyond the first layer. The curves are guides for the eye.

FIG. 12: (a) Reduced isosteric heat as a function of coverage for $\mathrm{Ne}, \mathrm{CH}_{4}$ and $\mathrm{Xe}$ at 
$\mathrm{T}^{*} \approx 0.75 . \epsilon_{g g}=35.6 \mathrm{~K}(\mathrm{Ne}), 161 \mathrm{~K}\left(\mathrm{CH}_{4}\right), 221 \mathrm{~K}(\mathrm{Xe})$.(b) Same as (a) but for $\operatorname{Ar}\left(\epsilon_{g g}=\right.$ $120 \mathrm{~K})$. The dots represent experimental results from Ref. 13.

FIG. 13: Heat capacity derived from classical simulations as a function of coverage for Ar at $\mathrm{T}=67.5 \mathrm{~K}$ (dashed curve) and $\mathrm{Kr}$ at $\mathrm{T}=71.25 \mathrm{~K}$ (full curve). 


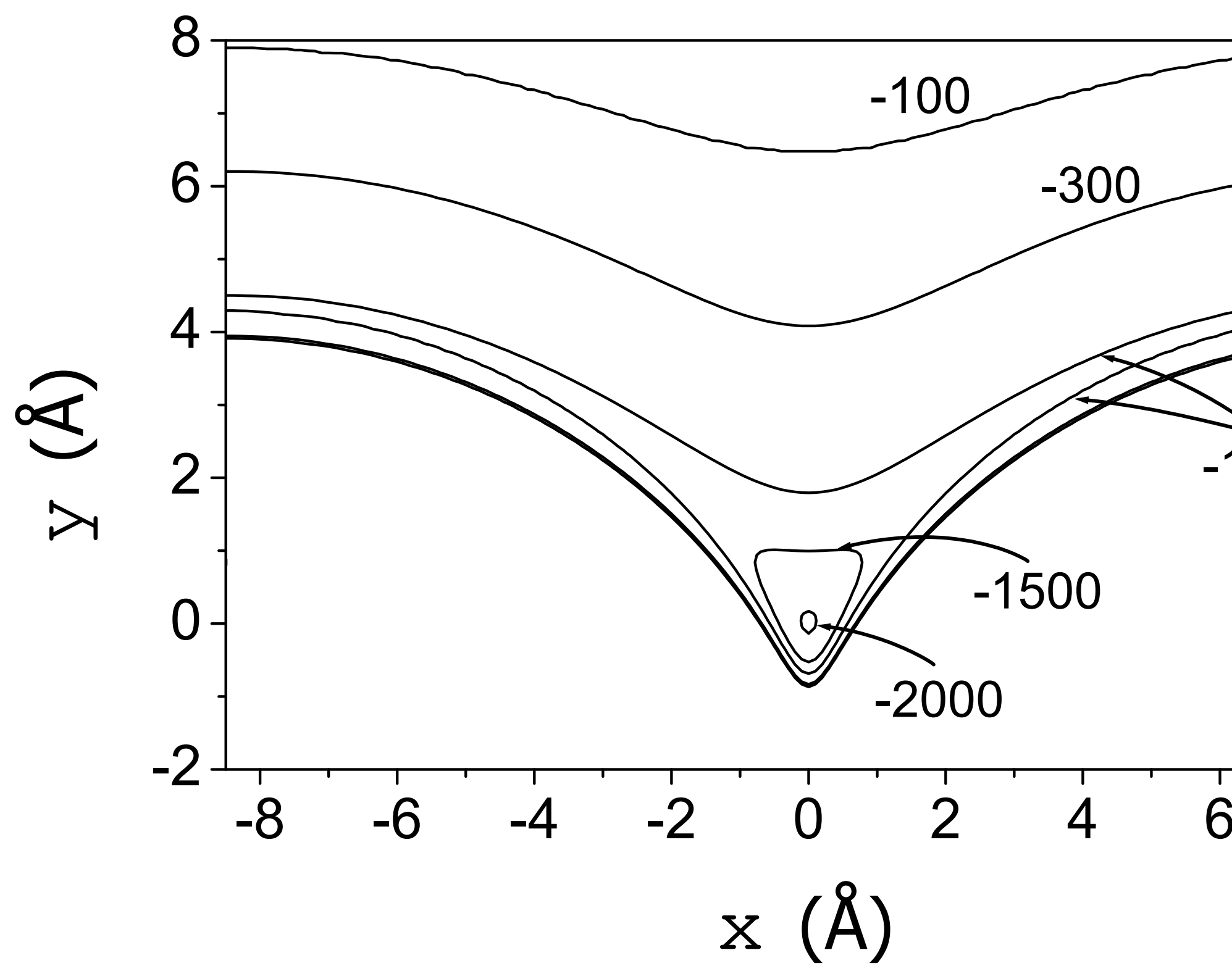



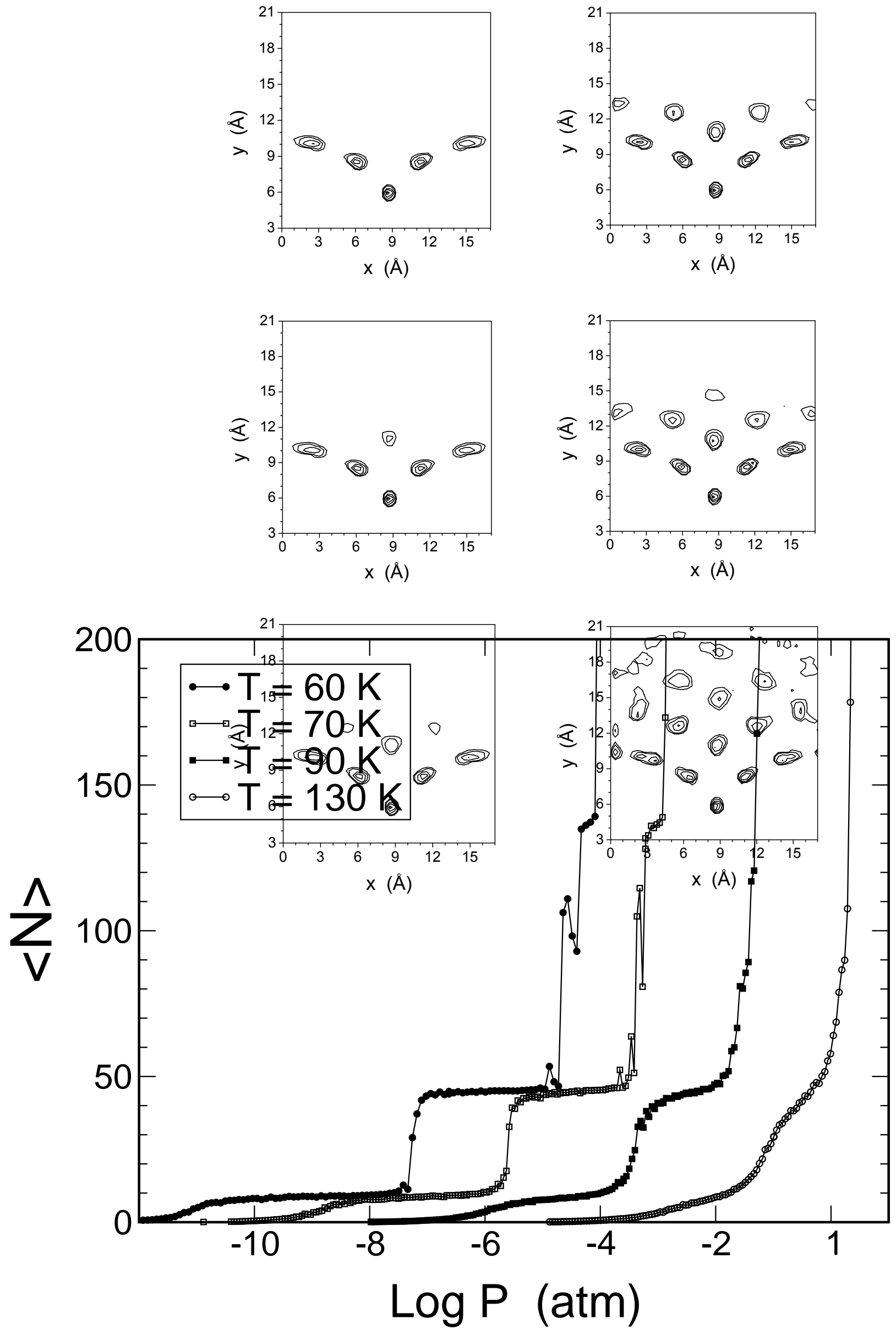

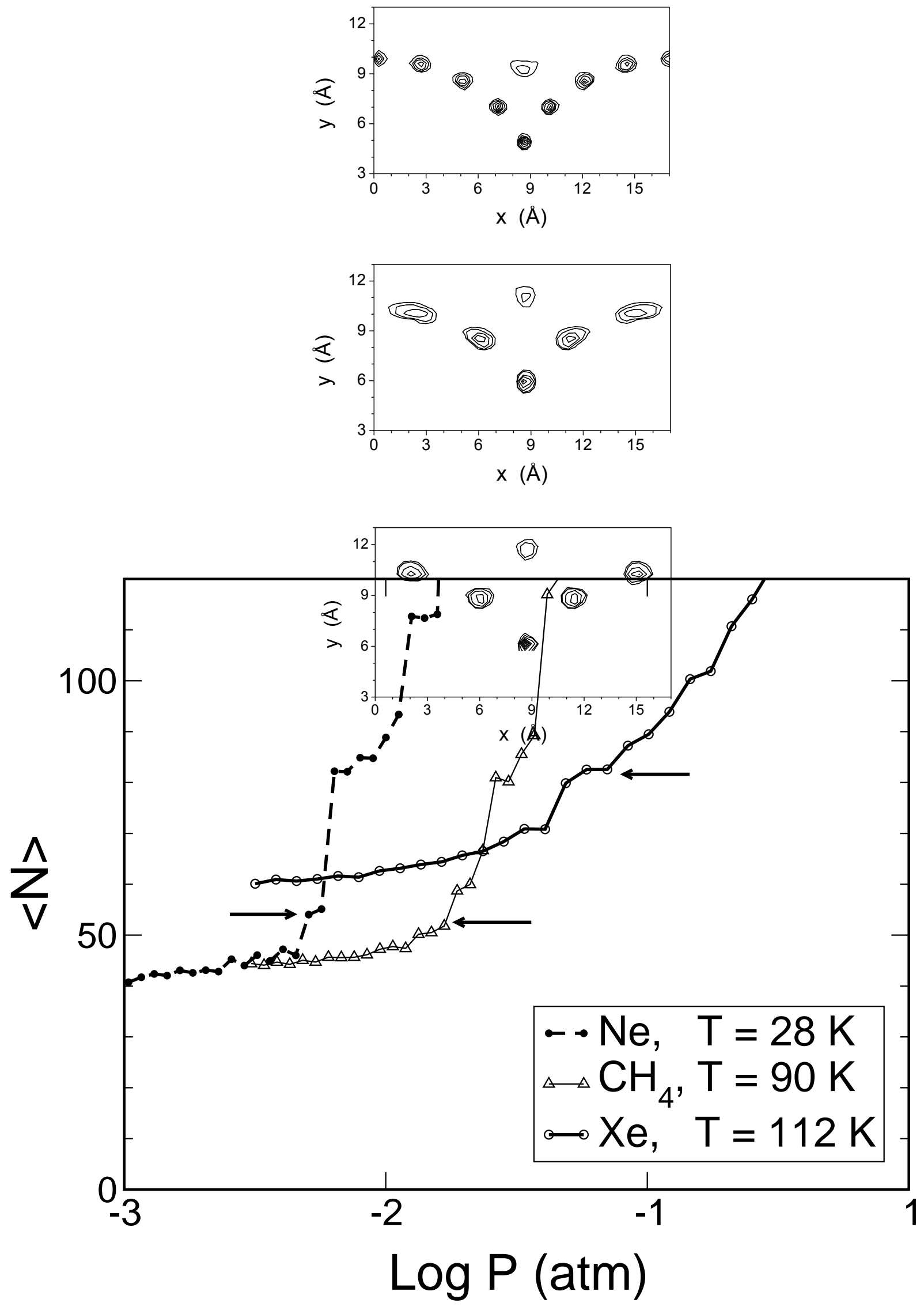